\title{
A Multiobjective Optimization Model for Flexroute Transit Service Design
}

\author{
Brian L. Smith and Michael J. Demetsky \\ University of Virginia
}

Priya K. Durvasula,

ConvaTec

\begin{abstract}
Flexroute transit (also referred to as route-deviation) is an innovative public transportation approach in which service is provided at fixed stops on a predetermined schedule, while also providing "on-demand" service to customers between the fixed stops. A significant challenge to the implementation of flexroute transit has been the complexity inherent in the design process. This research explored two of the key design parameters available to a transit planner: service zone size (the area between fixed stops where deviations are permitted), and slack time distribution (the method used to distribute among zones the total "slack" time built into the schedule to allow for deviations). It was found that the objectives of maximizing feasible deviations and minimizing unused slack time are conflicting and highly sensitive to the design parameters. To assist in the design process, the article formulates a multiobjective binary optimization model that can be used to develop an efficient frontier-guiding transit planners as they seek to explore design trade-offs.
\end{abstract}




\section{Introduction}

To address challenges resulting from such factors as low-density development and compliance with the Americans with Disabilities Act (ADA), many public transportation providers have turned to nontraditional service options such as flexroute transit (Rosenbloom 1996). In a flexroute system (also referred to as route-deviation), service is provided at fixed stops on a predetermined schedule, while also providing "on-demand" service to customers between the fixed stops. As seen in Figure 1, these characteristics suggest that flexroute transit is a hybrid of conventional fixed-route and paratransit services.

\section{Figure 1. Comparison of Flexroute, Paratransit and Fixed-Route Services}

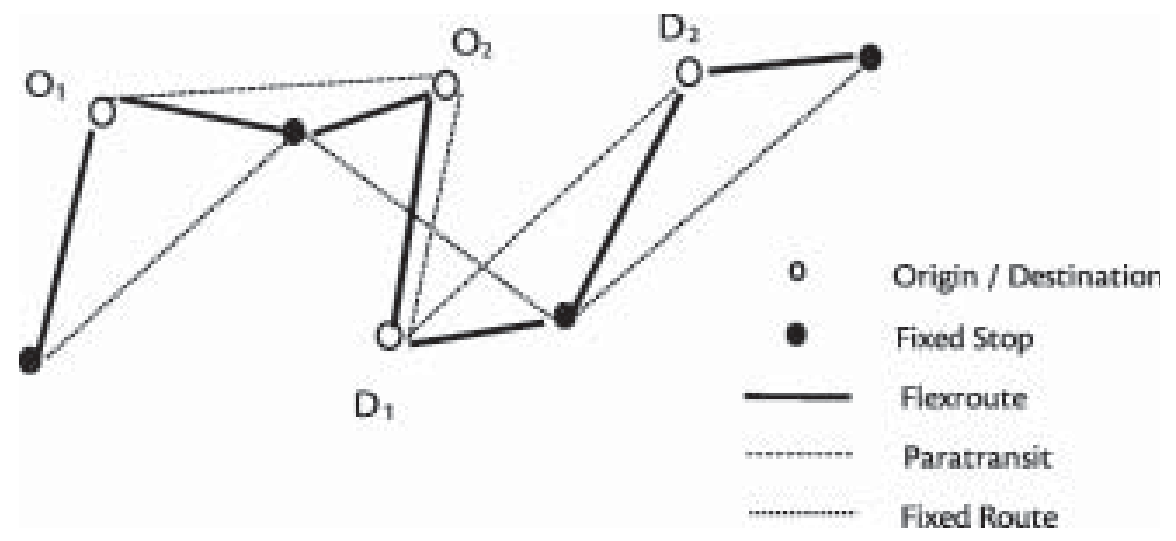

While there is significant interest in flexroute and other forms of demand responsive transit, transit providers are impeded by a relative lack of literature on the effective design and operation of such a service (Farwell 1998). This demonstrates the need to develop a methodology to aid in the design of an effective flexroute system. The research presented in this article addresses this need by using a threestep methodology:

1. Identifying the critical design parameters and outlining the relevant performance measures to evaluate the service under different designs

2. Performing a sensitivity analysis of the design parameters using a Geographic Information System (GIS)-based support system 
3. Developing a mathematical programming model to support optimal service design

In step 2, a GIS-based support system developed for scheduling and dispatching a flexroute service was used to evaluate the system performance given different design parameters. The GIS-based support system aides in routing and scheduling the on-demand aspect of the service within the bounds of the fixed-stop schedule constraints. The support system identifies the location of requested origins and destinations, determines the location within the on-demand service area, and calculates the time for the shortest deviated route to pick up and drop off the passenger. In addition, the support system keeps track of all committed rides and continually checks to ensure that any new ride will not prevent the transit vehicle from arriving at the fixed stops on schedule. A detailed description of the GIS-based support system can be found in Durvasula et al. (1998). For the purpose of this research, the GIS-based support system emulates the operation of a flexroute service, and therefore was used to conduct the sensitivity analysis.

In step 3, the results of the sensitivity analysis conducted in step 2 serve as inputs to a mathematical programming model. A multiobjective binary nonlinear programming model was developed to guide the design process that yielded the design parameter values for effective system performance. A description of the design methodology as well as the results of a case study are presented in this article. Before describing the service design methodology, a brief review of flexroute transit and a description of the study area used in this research are presented.

\section{Core Flexroute Concepts}

The following are the fundamental terms, definitions, and assumptions concerning flexroute transit considered in this research.

- A service zone is defined as the region between a pair of two consecutive fixed stops on a given route. Each zone is given a unique identification number based on the associated route and the fixed stops that bound the region. For example, Zone 1 is the service area between the fixed stops 1 and 2; Zone 2 is the area between fixed stops 2 and 3 and so on. This definition is used to locate the position of the origin and destination locations of a trip request with respect to the fixed stops.

- The transit vehicle will make mandatory stops at the fixed stops and adhere to the constraint of arriving on schedule at these stops. 
- The total time needed to travel from one end of the route to the other end is fixed. This time includes the built-in slack in the schedule for deviating to pick up/drop off on-demand passengers in service zones.

\section{Study Area}

To provide a context for this effort, the research team worked with Hampton Roads Transit (HRT), an agency that was considering the introduction of flexroute service in the Peninsula region of Virginia (the region anchored by the cities of Hampton and Newport News). In addition to traditional fixed-route service, HRT also provides paratransit services, HandiRide, for the disabled under ADA mandates.

Two of HRT's existing fixed routes were chosen to serve as the "routes" for flexroute service in this study. The two routes, Routes 10 and 11, were chosen in consultation with HRT management and were "conceptually" modified to enable flexroute service. Fixed stops with low ridership were eliminated for the two routes. Each retained up to a maximum of five major fixed stops. Additionally, the schedules at these stops were changed to incorporate some slack time between the stops to allow for deviation. A methodology described in Welch et al. (1991) was used to realign the fixed routes.

\section{Step 1: Design Parameters and Performance Measures}

The critical design parameters and relevant performance measures identified for flexroute service are described in this section.

\section{Flexroute Design Parameters}

The two important design parameters identified and studied in this research are: service zone size and the slack time distribution method. These are discussed below.

\section{Service Zone Size}

The service zone area of a flexroute service defines how far away from the standard route a vehicle may deviate to pick up or drop off a passenger. The service area defines the maximum feasible deviation distance. It does not imply that everyone within the zone is guaranteed a deviated trip. At times, previously committed rides will force a requested trip from within a service zone to be denied.

By virtue of the definition of a service zone, the total number of zones for a given route will always be one less than the total number of fixed stops. Prima- 
rily due to a lack of more complete guidance, most transit systems have limited flexroute service zones to a $3 / 4$ mile distance on either side of the nominal fixed route. However, due to the myriad of factors that differ from zone to zone, such as potential ridership, street network connectivity, population density etc., a service area of $3 / 4$ mile on either side of the nominal fixed route may not be universally ideal. This warrants the need to analyze the effect of different service zone sizes on the performance of a route deviation system. Three service zones' distances were considered in this research: $400 \mathrm{~m}, 800 \mathrm{~m}$, and $1,200 \mathrm{~m}(1 / 4,1 / 2$, and $3 / 4$ mile).

\section{Slack Time Distribution}

Clearly, the total route running time for a flexroute system must be greater than the sum of the direct travel times between fixed stops. Otherwise, no deviations would be possible in the system. This "extra" time that is built into the schedule is referred to as slack time.

\section{Given:}

- the total nonstop travel time or running time between two consecutive fixed stops (F)

- the total running time of the entire route in minutes ( $T$, which for this study is 60 minutes)

The total slack time for a route can be computed from the relation: $S=(T$ $F)$. Clearly, there is a desire to limit slack time to prevent "idle" vehicles in the absence of flexroute requests. The slack time built into each route (in each direction, i.e. inbound/outbound) for the purposes of this study ranged from 5 to 15 minutes, with an average slack time of 8 minutes. The next challenge in flexroute service design is to determine the best way to distribute the slack time among a route's service zones. In this investigation, two approaches to slack time distribution were considered:

1. Slack time distribution as a weighted average of the nonstop travel time between the two fixed stops of a zone (SDWR), and

2. Slack time distribution as a weighted average of the total number of origins and destinations of ADA certified trips from HandiRide logs (SDNP) 


\section{System Performance Measures}

The influence of the design parameters was investigated using system-specific performance measures. The two performance measures identified and selected in this research, and the reasons for choosing them, are described below.

\section{System Perspective}

The maximum allowable or feasible (given all schedule constraints) deviations per hour was chosen as the performance measure from the perspective of a flexroute provider. This measure was chosen because it measures the viability of a flexroute service in terms of the number of requests/calls that can be served within the bounds of fixed-stop schedules. That is, it measures the maximum supply of service that an operator can provide within the given constraints of the system. Once the limit of the supply is known, it can be compared against the demand for flexroute service to see if the demand can be met adequately. The objective of a flexroute provider would be to maximize the value of this performance measure.

\section{Customer Perspective}

The cumulative unused slack time remaining at all fixed stops per route was chosen as the performance measure from the perspective of a customer using the flexroute service. Although the additional dwell time does not affect the estimated time of arrival at the customer's destination, it measures the "perceived" inconvenience of a rider in the event of a transit vehicle arriving at the fixed stop earlier than the scheduled time. Early arrivals at scheduled stops are always discouraged in fixed-route services, and provide an important determinant in transit level of service and transit network design (Gray and Hoel 1992). The objective of a flexroute transit provider would be to minimize this value for each service zone. 
The two performance measures were also chosen because they are universally applicable in measuring the level of service provided by flexroute transit from the perspectives of the provider and customer.

\section{Step 2: Sensitivity Analysis of Design Parameters}

Table 1 illustrates the sensitivity analysis scenarios and performance measures used to evaluate each scenario. A combination of each tested design parameter value yields a total of 6 possible scenarios.

\section{Table 1. Sensitivity Analysis Scenarios and Performance Measures}

\begin{tabular}{|c|l|}
\hline Design Parameter & \multicolumn{1}{|c|}{ Scenarios } \\
\hline $\begin{array}{c}\text { Service zone size } \\
\text { (Distance from the route) }\end{array}$ & $\begin{array}{l}\text { 1. } 400 \mathrm{~m}(1 / 4 \text { mile) } \\
\text { 2. } 800 \mathrm{~m}(1 / 2 \mathrm{mile}) \\
\text { 3. } 1,200 \mathrm{~m}(3 / 4 \mathrm{mile})\end{array}$ \\
\hline $\begin{array}{c}\text { Slack Time (ST) } \\
\text { distribution }\end{array}$ & $\begin{array}{l}\text { 1. ST distributed by weighted average of running } \\
\text { time between stops (SDWR) }\end{array}$ \\
\hline
\end{tabular}

The analysis was conducted using the GIS-based support system described earlier to emulate the system performance under each scenario. For the network analysis, the vehicle dwell time values at the fixed and deviated stops were estimated based on common methods available in the literature (see Durvasula et al., 1998 for details). For each service zone, a random number generator was used to randomize the order in which persons were served (emulating different request patterns). Requests for deviations for each zone were picked from a list of HandiRide addresses that were within that particular service zone. After randomizing the order, the requests were added one after the other from the list to the support system, each time checking whether the additional request was feasible within the flexroute constraints. If the addition of a point was not feasible, it was dropped 


\section{Table 2. Effect of Slack Time Distribution Methods on the Number of Feasible Deviations}

\begin{tabular}{|c|c|c|c|c|c|c|}
\hline \multirow{2}{*}{} & \multicolumn{2}{|c|}{$400 \mathrm{~m}$ Zone Distance } & \multicolumn{2}{c|}{$800 \mathrm{~m}$ Zone Distance } & \multicolumn{2}{l|}{$1,200 \mathrm{~m}$ Zone Distance } \\
\cline { 2 - 8 } & SDWR & SDNP & SDWR & SDNP & SDWR & SDNP \\
\hline Zone 1 & 6.00 & 6.88 & 6.00 & 5.88 & 5.5 & 5.3 \\
\hline Zone 2 & 2.91 & 6.36 & 2.50 & 5.71 & 2.38 & 5.38 \\
\hline Zone 3 & 5.57 & 3.86 & 5.80 & 5.00 & 5.83 & 4.5 \\
\hline Route 11 total & 14.48 & 17.10 & 14.30 & 16.59 & 13.71 & 15.18 \\
\hline Zone 4 & 1.50 & 2.00 & 1.50 & 1.00 & 1.4 & 1 \\
\hline Zone 5 & 2.00 & 2.00 & 1.50 & 2.38 & 3.5 & 2.9 \\
\hline Zone 6 & 3.80 & 3.80 & 3.38 & 3.38 & 1.42 & 1.83 \\
\hline Zone 7 & 9.00 & 8.00 & 6.06 & 6.06 & 5.4 & 6.8 \\
\hline Route 10 total & 16.30 & 15.80 & 12.64 & 12.80 & 11.72 & 12.53 \\
\hline Overall totals & 30.78 & 32.90 & 26.74 & 29.39 & 25.43 & 27.71 \\
\hline
\end{tabular}

from the list of feasible points. Several iterations were performed to exhaustively include all the different orders of requests.

\section{Results of the Sensitivity Analysis}

Tables 2 and 3 summarize the results of the sensitivity analysis. The numbers presented in the two tables are the mean values for the two performance measures obtained at the end of all iterations for each zone.

The experiments indicate that the performance measures are indeed quite sensitive to the design parameter values. Upon a close examination of the results above, one can see that for Route 11, a zone distance of $400 \mathrm{~m}$ with a slack time distribution method of SDNP will yield the largest number of feasible deviations, whereas for the additional dwell-time performance measure a zone distance of $800 \mathrm{~m}$ with the same slack time distribution method yields the best value. Hence, the "best" design parameter combination is not the same under the two performance mea- 


\section{Table 3. Effect of Slack Time Distribution Methods on Additional Dwell Time (in seconds) at the Fixed Stops}

\begin{tabular}{|c|c|c|c|c|c|c|}
\hline \multirow{2}{*}{} & \multicolumn{2}{|c|}{$400 \mathrm{~m}$ Zone Distance } & \multicolumn{2}{c|}{$800 \mathrm{~m}$ Zone Distance } & \multicolumn{2}{c|}{$1,200 \mathrm{~m}$ Zone Distance } \\
\cline { 2 - 7 } & $50 W \mathrm{~N}$ & SDNP & $50 \mathrm{WR}$ & SDNP & SDWR & SDNP \\
\hline Zone 1 & 52.96 & 53.46 & 52.96 & 1.46 & 46.11 & 5.39 \\
\hline Zone 2 & 41.43 & 27.27 & 31.38 & 55.78 & 30.21 & 54.92 \\
\hline Zone 3 & 263.49 & 54.87 & 110.64 & 39.79 & 76.56 & 40.35 \\
\hline Route 11 total & 357.88 & 135.60 & 194.98 & 97.03 & 152.88 & 100.67 \\
\hline Zone 4 & 38.78 & 59.42 & 38.78 & 20.89 & 31.02 & 20.89 \\
\hline Zone 5 & 35.93 & 35.93 & 63.16 & 63.16 & 15.28 & 25.4 \\
\hline Zone 6 & 11.05 & 71.05 & 39.52 & 57.02 & 33.66 & 41.06 \\
\hline Zone 7 & 54.11 & 150.11 & 30.69 & 30.69 & 39.14 & 29.18 \\
\hline Route 10 total & 139.87 & 316.51 & 172.16 & 171.76 & 119.1 & 116.53 \\
\hline Overall totals & 497.75 & 452.11 & 367.14 & 268.79 & 271.98 & 217.2 \\
\hline
\end{tabular}

sures for a given route. This is further complicated by the fact that, unlike the assumption made in the sensitivity analysis, the buffer distance design parameter is actually zone-specific while the slack time distribution method is route-specific.

Thus, it is evident that the design of a flexroute service is a complex, multiobjective problem. Attempting to develop a "near"-optimal design manually, particularly as the number of routes and zones increase, is practically infeasible. Therefore, the research team explored the use of automated optimization techniques as a means to support the design of flexroute systems. 


\section{Step 3: Multiobjective Nonlinear Choice Model}

The problem of identifying the "best" values of design parameters per service zone and route in a flexroute system has been formulated as a multiobjective nonlinear integer-programming model. Furthermore, the model constrains all decision variables as binary (0-1) integer variables. As such, the model is an "assignment" or "coded" model that searches for the optimal matching or pairing of objects of two distinct types under assignment constraints (i.e., each object of each set may be paired only once). The multiobjective aspect of the model is addressed by identifying Pareto optimal or efficient points and using them to construct an "efficient frontier" (Rardin 1997).

\section{Binary Nonlinear Program: Model Formulation}

The variables used in formulating the objective functions and constraints of the nonlinear model are defined in Table 4. It is important to carefully consider the design parameter values that must be identified for a given route and its service zones: the slack time distribution method (e.g., SDWR and SDNP) for a route, and service zone sizes (e.g., $400 \mathrm{~m}, 800 \mathrm{~m}$, and $1,200 \mathrm{~m}$ ) for each zone within a route. Clearly, there is a distinction in the assignment of the two design parameters. Namely, slack time distribution is a route-specific design parameter while service zone size is a zone-specific design parameter. This distinction leads to the following assignment constraint: performance measure values for zones within a given route cannot be chosen from two different slack time distribution methods as it would not be practicable.

The binary nonlinear model is presented by equations 1 through 6 . The decision variables are discrete and are represented by $X_{r i j}, S_{r l}$, and $S_{r 2}$ and the model is rendered nonlinear by the two objective functions (which must be computed using the GIS-based support system). Equations 1 and 2 represent the two objective functions, where (1) minimizes the additional dwell time (or the unused slack time) and (2) maximizes the number of feasible deviations performance measure. The index ranges in the objective functions assure that each combination of service zone and design parameter is considered only once. Rather than utilize a decision variable for each combination of design parameters for each zone, an additional set of decision variables representing each slack time distribution method is used. This incorporates the route-specific nature of the slack time distribution method. 


\section{Table 4. Variables Used in the Integer Programming Model}

\begin{tabular}{|c|c|}
\hline Variables & Descripunan \\
\hline & Derition Horimbles in the Monteis \\
\hline$x_{\text {w }}$ & $\begin{array}{l}\text { The nonlinear } 0-1 \text { integer decisian variable for the } \mathrm{r}^{\mathrm{B}} \text { route, } \mathrm{i}^{\mathrm{B}} 2 \mathrm{zone}, \mathrm{j}^{\mathrm{H}} \\
\text { buffer distance }\end{array}$ \\
\hline$s_{n}$ & $\begin{array}{l}\text { The nonlinear } 0-1 \text { imteger decisian variable for } \mathrm{r}^{\mathrm{*}} \text { route using Slack time } \\
\text { distribution method } 1\end{array}$ \\
\hline $\boldsymbol{S}_{n}$ & $\begin{array}{l}\text { The nonlinear 0-1 imleger decision variable for the } \mathrm{r}^{ }{ }^{\mathrm{N}} \text { roule usihe Slack } \\
\text { time distribution method } 2\end{array}$ \\
\hline & Inputs or Knoun Vabiues \\
\hline$D_{\text {nent }}$ and $N_{\text {nes at }}$ & 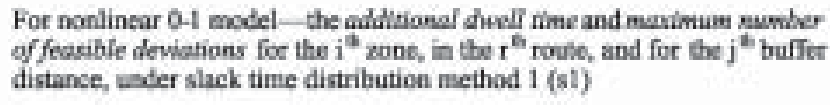 \\
\hline$D_{\text {nos al }}$ and $N_{\text {nat }}$ & 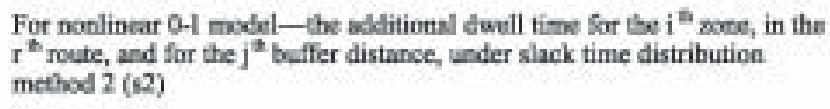 \\
\hline$N_{x}$ & The total number of zones in the system \\
\hline$z$ & Number of zones in the $\mathrm{r}^{\mathrm{in}}$ route \\
\hline$N_{R}$ & Totsl mumber of routes in the system. \\
\hline$N_{2 x}$ & Average sumber of zones per route in a system \\
\hline$N_{\mathbf{b}}$ & Totsi number of buffer disances \\
\hline$s_{\mathrm{s}}$ & Total mumber of methods for distributing the slack time \\
\hline
\end{tabular}




$$
\begin{aligned}
& \text { Minimize } \sum_{r=1}^{N_{n}} \sum_{i=1}^{Z_{r}} \sum_{j=1}^{N_{k}} x_{r i j}\left[\left(D_{r \tilde{r}, s_{1}} s_{r 1}\right)+\left(D_{r i j, s_{2}} s_{r 2}\right)\right] \\
& \operatorname{Maximize} \sum_{r=1}^{N_{n}} \sum_{j=1}^{Z_{k}} \sum_{j=1}^{N_{k}} x_{r j}\left[\left(N_{r j, s_{1}} s_{r 1}\right)+\left(N_{r i j} s_{2} s_{r 2}\right)\right]
\end{aligned}
$$

s.t.

$$
\begin{aligned}
& \sum_{j=1}^{N_{k}} x_{r y}=1 \text { for all } r \text { and } i \\
& s_{r 1}+s_{r 2}=1 \text { for all } r \\
& \sum_{r=1}^{N_{n}} \sum_{i=1}^{z_{r}} \sum_{j=1}^{N_{n}} x_{r \ddot{r}}=N_{z} \\
& x_{r i j}, s_{r 1}, s_{r 2}=0 \text { or } 1 \text { for all } r, i, j
\end{aligned}
$$

The two objective functions represented by equations (1) and (2) have been formulated for the case considering only two slack time distribution methods. The formulation could be extended to a general case where one considers more than two methods, i.e., $S_{n}>2$, and the objective functions may be rewritten as:

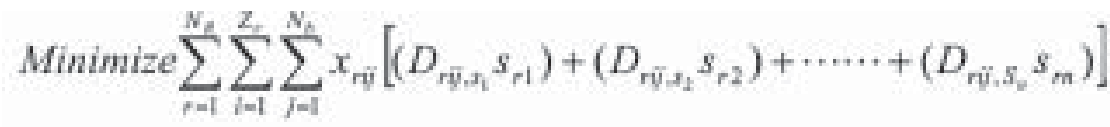

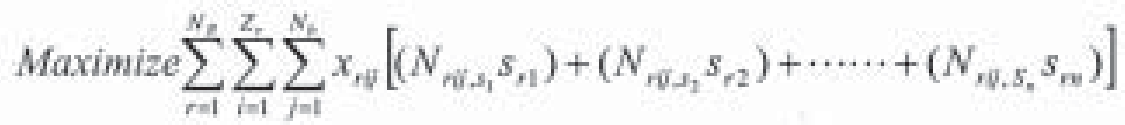

The constraint modeled by equation (3) ensures that a single zone distance is assigned to each service zone in the system. Equation (4) is important in that it ensures that only one slack time distribution method is chosen for each route. 
That is, this constraint accounts for the route specific nature of the slack time distribution design parameter. Constraint (5) satisfies the condition that the sum of all the decision variables' values must equal the total number of zones in the system. This implies that each zone must be assigned a zone distance and a slack time distribution method. Finally, equation (6) incorporates the 0-1-integer constraint for the binary decision variables. The objective functions of the model have only second order terms, and all the constraints are linear, thus making it a quadratic program.

The algorithm used to solve the nonlinear integer program is the generalized reduced gradient (GRG) method developed by Lasdon et al. (1978). This method is known to be susceptible to converging to a local optimum. To improve the likelihood that the solution obtained was globally optimal, this limitation was addressed by frequently changing the initial values of the decision variables (known as the "multistart" procedure). The global optimum was determined from the range of the objective values obtained from all the trials.

\section{Results: Two Objectives Considered Separately}

The model was solved first by considering each objective independently, before considering the multiobjective formulation. Although only one objective function was optimized in each model, the resulting values of the second objective are also reported in Table 5.

\section{Table 5. Local and Global Optima for Each Objective Function Solved Separately}

\begin{tabular}{|c|c|}
\hline \multicolumn{2}{|c|}{$\begin{array}{c}\text { Model 1: } \\
\text { Maximize Na. of Deviations } \\
\text { (1st Obj.) }\end{array}$} \\
\hline 1st Obj. Value & 2nd Obj. Value \\
\hline 34.94 & 426.56 \\
\hline
\end{tabular}

\begin{tabular}{|c|c|}
\hline \multicolumn{2}{|c|}{ Model 2: } \\
Minimize Additional Dwell \\
Time (2nd Obj.)
\end{tabular}


The decision variable values that result in the optimal objective function values of 34.94 for the first objective, and 156.56 for the second objective, solved separately, are presented in Table 6 . In the case of the first objective (i.e., maximize number of deviations), the slack time distribution method chosen for each route is SDNP. For the second objective, SDNP was chosen for Route 11 while SDWR was chosen for Route 10. The choice of SDWR for Route 10 of the second objective can be understood by comparing the totals in Tables 2 and 3 for SDWR and SDNP for Route 10 (Zones 4 through 7). Notice that the total additional dwell time for Route 10 under 1,200 and $800 \mathrm{~m}$ zone sizes for SDWR is only slightly higher than the totals for SDNP. For a zone size of $400 \mathrm{~m}$, however, the total additional time under SDWR is much lower than for SDNP (139.87 v. 316.51). For this reason the minimize dwell time objective SDWR is preferred over SDNP. This illustrates the effectiveness of the optimization model in identifying the combination of design parameters that yield the best design. Additionally, the results in Table 6 indicate that zones within a given route may indeed need to have different sizes in order to improve the flexroute service level. This substantiates the assumption that there is a need for an automated optimization model to assist in the search for the best design parameters given the zone-to-zone variability within a single route.

\section{Results: Efficient Frontier-Multiobjective Problem}

Based on the results obtained from solving the optimization model considering the objective functions independently, it is clear that the objectives are conflicting. That is, achieving a larger number of feasible deviations results in a longer additional dwell time. This "conflict" requires a decision-maker to consider trade-offs between these objectives. The designs identified above represent extremes; thus, there is a need to carefully consider possible "trade-off" designs between the extremes. This is referred to as developing the efficient frontier.

The notation [ND, ADT] is used to represent the pair of objective function values resulting from a particular flexroute design, where ND stands for number of feasible deviations (to be maximized) and ADT for additional dwell time (to be minimized). The feasible range of these values are known from solving the two objectives separately, and are [34.94, 426.56] and [30.19, 156.56]. To construct the efficient frontier, the second objective (i.e., additional dwell time) was incorporated into the 0-1 INLP model as an inequality constraint with a new parameter a (where the values of a 1 [156.56, 426.56], the limits of the range of values for the 
Table 6. Actual Performance Measure Values Selected by the Model for the Global Optimum

\begin{tabular}{|c|c|c|c|c|c|c|}
\hline \multicolumn{4}{|c|}{ L. Maximizing No. of Devlations } & \multicolumn{3}{|c|}{ IL. Minimizing Add. Dwell Time } \\
\hline \multicolumn{4}{|c|}{ No. of Deviations } & \multicolumn{3}{|c|}{ Na of Deviations } \\
\hline & $1,200 \mathrm{~m}$ & $800 \mathrm{~m}$ & $400 \mathrm{~m}$ & $1,200 \mathrm{~m}$ & $800 \mathrm{~m}$ & $400 m$ \\
\hline Zone 1 & - & . & 6.88 & - & 5.88 & - \\
\hline Zone 2 & - & - & 6.36 & - & - & 6.36 \\
\hline Zone 3 & - & 5.00 & . & . & 5.00 & - \\
\hline Zone 4 & - & - & 2.00 & 1.40 & - & - \\
\hline Zone 5 & 2.90 & - & - & 3.50 & - & - \\
\hline Zone 6 & $=$ & - & 3.80 & - & - & 200 \\
\hline Zone 7 & - & - & 8.00 & - & 6.06 & - \\
\hline \multicolumn{4}{|c|}{ Additional Dwell Time (seconds) } & \multicolumn{3}{|c|}{ Additional Dwell Time (seconds) } \\
\hline & $1,200 \mathrm{~m}$ & $800 m$ & $400 \mathrm{~m}$ & $1,200 \mathrm{~m}$ & $800 m$ & $400 \mathrm{~m}$ \\
\hline Zone 1 & . & - & 53.46 & . & 1.46 & . \\
\hline Zone 2 & - & - & 27.27 & - & - & 27.27 \\
\hline Zone 3 & - & 39.79 & - & - & 39.79 & . \\
\hline Zone 4 & $=$ & - & $\$ 9.42$ & 31.02 & $=$ & . \\
\hline Zone 5 & 25.40 & - & - & 15.28 & - & - \\
\hline Zone 6 & - & - & 71.05 & - & - & 11.05 \\
\hline Zone 7 & - & - & 150.11 & - & 30.69 & - \\
\hline
\end{tabular}


Journal of Public Transportation,Vol. 6, N o. 1, 2003

second objective function). The 0-1 INLP model formulation with the second objective introduced as a constraint is given as follows:

$$
\begin{aligned}
& \operatorname{Maximize} \sum_{r=1}^{N_{k}} \sum_{i=1}^{Z_{r}} \sum_{j=1}^{N_{k}} x_{r \bar{y}}\left[\left(N_{r \bar{y} J_{1}} s_{r 1}\right)+\left(N_{r i \bar{y}, s_{2}} s_{r 2}\right)\right] \\
& \text { s.t. } \\
& \sum_{r=1}^{N_{k}} \sum_{i=1}^{Z_{i}} \sum_{j=1}^{N_{k}} x_{r y}\left[\left(D_{r i j, s_{1}} s_{r j}\right)+\left(D_{r i j, s_{2}} s_{r 2}\right)\right] \leq \alpha \\
& \sum_{i=1}^{N_{1}} x_{r i j}=1 \text { for all } r \text { and } i \\
& s_{, 1}+s_{, 2}=1 \text { for all } r \\
& \sum_{r=1}^{N_{m}} \sum_{j=1}^{Z_{r}} \sum_{j=1}^{N_{k}} x_{r i j}=N_{z} \\
& x_{r j}, s_{r 1}, s_{r 2}=0 \text { or } 1 \text { for all } r, i, j
\end{aligned}
$$

The above model was solved using different feasible values of a to construct the efficient frontier, which is displayed in Figure 2. 


\section{Figure 2. Efficient Frontier for the Multiobjective Optimization Problem}

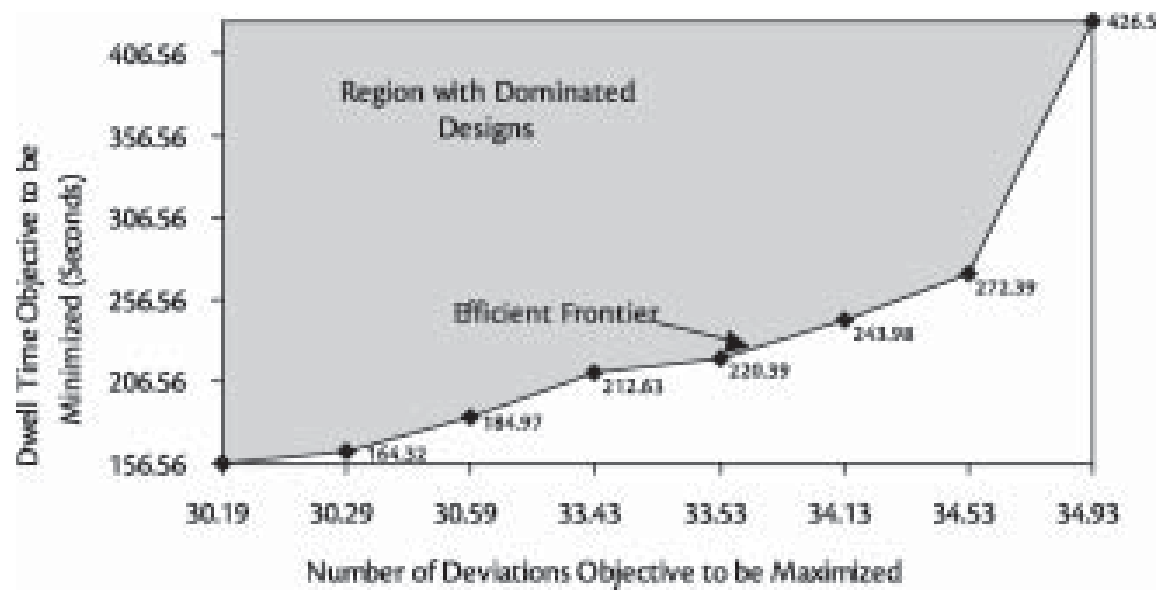

An important insight gained from Figure 2 is that over the efficient frontier, the values of the number of feasible deviations objective vary over a smaller range $(30.19,34.93)$ when compared to the range of the dwell time objective (156.56, 426.5). This indicates that the number of feasible deviations objective would not be considerably degraded (a change of only 4 ) if the solution for the best value of the additional dwell time objective were to be selected (i.e., 156.56 seconds). Whereas, if the best value for the number of deviations objective is selected, then the corresponding dwell time objective will be 426.5 seconds, nearly 3 times the lowest value of 156.56 seconds. Therefore, based on the results for this particular case study, it would be recommended that the design parameter values for the solution with the objective values, $(30.19,156.56)$, be used for the flexroute service.

\section{Condusions}

While there is significant interest in the concept of flexroute transit service, a significant implementation impediment is the complexity inherent in designing the service. This research identified and explored key flexroute transit design parameters: service area and slack time distribution. Specifically, the results of this effort indicate that for a constant slack time, as the area of the service zone is increased, the number of feasible deviations that can be accommodated decreases. However, the additional dwell time, or the excess slack time left over, increases with the 
reduction in service area. Thus, the objectives of increasing the number of feasible deviations and simultaneously reducing the excess slack time are conflicting. For this reason, and the large number of design options available when designing multiple route, multiple zone flexroute service, a multiobjective binary optimization model was formulated to (1) choose the "best" combination of design parameter values for each of the two objectives, and (2) determine the relative change in the values of the two objectives by constructing the efficient frontier. The model is the final step in the service design methodology and will be very useful for a system with a large number of routes and zones. Finally, additional design parameter values can be introduced in the model to determine their effect on design of route service. 


\section{References}

Durvasula, P. K., B. L. Smith, R. E. Turochy, S. C. Brich, and M. J. Demetsky. 1998. Peninsula Transportation District Commission route deviation feasibility study. VTRC Report No. 99-R11. Charlottesville, VA: Virginia Transportation Research Council.

Farwell, R. G. 1998. Evaluation of Omnilink demand-driven transit operations: Flex-route services. Transportation Quarterly 52 (1).

Gray, G. E., and L. A. Hoel, eds. 1992. Public transportation. Englewood Cliffs, NJ: Prentice-Hall, Inc.

Lasdon, L. S., A. Warren, A. Jain, and M. Ratner. 1978. Design and testing of a generalized reduced gradient code for mnlinear programming. ACM Transactions on Mathematical Software 4 (1):34-50.

Rardin, R. L. 1997. Optimization in operations research. New Jersey: Prentice Hall.

Rosenbloom, S. 1996. Service routes, route deviation, and general public paratransit in urban, suburban, and rural transit systems. Project Report AZ-26-7000. Washington, DC: U.S. Department of Transportation, Federal Transit Administration.

Welch, W., R. Chisholm, D. Schumacher, and S. R. Mundle. 1991. Methodology for evaluating out-of-direction bus route segments. Transportation Research Record 1308, pp. 43-50.

\section{About the Authors}

BRIAN L. SMITH (briansmith@virginia.edu) is an assistant professor of civil engineering at the University of Virginia. He is the university director of the Smart Travel Laboratory where he conducts research in the area of intelligent transportation systems.

Michael J. Demetsky (mjd@virginia.edu) is professor and chair of civil engineering at the University of Virginia. He is the former director of the Center for Transportation Studies and conducts research in the area of transportation planning and operations. 
Journal of Public Transportation,Vol. 6, N o. 1, 2003

Priya K. Durvasula (priya.durvasula@bms.com) obtained her Ph.D. in civil engineering from the University of Virginia. She is currently a principal analyst at ConvaTec. 